\title{
Fractionated stereotactic radiotherapy: an interesting alternative to stereotactic radiosurgery in acromegaly
}

\author{
Emmanuelle Kuhn ${ }^{1,2,3} \cdot$ Philippe Chanson ${ }^{1,2,3}$
}

Received: 23 September 2015 / Accepted: 30 September 2015/Published online: 13 October 2015

(C) Springer Science+Business Media New York 2015

To the Editor,

Although it is almost always due to a benign tumor, acromegaly remains a severe disease responsible for disabling symptoms, comorbidities, and shortened lifespan if left untreated [1]. Neurosurgery is a very effective first-line treatment for pituitary microadenomas $(<10 \mathrm{~mm})$, generally inducing remission [2]. In contrast, transsphenoidal neurosurgery is less effective for macroadenomas, particularly when invasive, which is the case of most patients with acromegaly. Somatostatin analogs, dopamine agonists, and/or a GH receptor antagonist can improve postsurgical disease control in such patients [3], but medical treatment has several drawbacks. In particular, it is expensive and necessitates lifelong injections. In addition, these drugs are not fully effective, even when used in combination, and their use is sometimes limited by severe and persistent adverse effects. Thus, in a number of cases, particularly when the stepwise approach based on surgery and medical therapy fails to control acromegaly, when drug tolerance is poor, and/or when the patient is weary of potentially lifelong medical treatment, it is legitimate to propose radiotherapy [4].

Philippe Chanson

philippe.chanson@bct.aphp.fr

1 Service d'Endocrinologie et des Maladies de la Reproduction, Assistance Publique-Hôpitaux de Paris, Hôpital de Bicêtre, 94275 Le Kremlin-Bicêtre, France

2 Unité Mixte de Recherche-S1185, Univ Paris-Sud, Université Paris Saclay, Faculté de Médecine Paris-Sud, 94276 Le Kremlin Bicêtre, France

3 Unité 1185, Institut National de la Santé et de la Recherche Médicale, 94276 Le Kremlin Bicêtre, France
Radiotherapy techniques have evolved over the last 20 years. Radiation can be delivered either in a single session by stereotactic radiosurgery (SRS), or fractionated during multiple sessions. Radiosurgery, performed in a single session after 3D localization of the culprit lesion, induces remission in a large proportion of patients with small adenoma remnants distant from the optic chiasm [5]. Various SRS modalities are available, and radiation can be delivered either as photons (Gamma Knife, Linac, CyberKnife) or charged particles (protons). SRS is used to deliver 20-25 Gy in one or a few sessions. However, radiosurgery is unsuitable for patients with large invasive tumors or even small adenomas close the optic nerves or chiasm [6, 7]. Alongside "conventional" fractionated radiotherapy, new radiotherapy techniques have been developed with the aim of reducing irradiation of healthy brain tissue surrounding the tumor. Fractionated stereotactic radiotherapy (FSRT) is a hybrid modality which, as its name implies, combines stereotactic localization with fractionated therapy, administered as 3D-conformal, intensity-modulated or proton beams. In acromegaly, FSRT is used to deliver an average total dose of $50 \mathrm{~Gy}$ in about 25 daily sessions. The choice between the two current methods (radiosurgery and FSRT) depends both on the size of the tumor and on its vicinity to the optic apparatus.

FSRT is generally preferred for large tumors (generally more than $3 \mathrm{~cm}$ ) and for adenomas located more than 3-5 $\mathrm{mm}$ from the optic chiasm [7]. Its efficacy and tolerability have so far only been evaluated in a few studies with short follow-up [8-11]. The paper by Diallo AM et al. in this issue of Endocrine provides valuable information on the long-term effects of FSRT on hormonal status and tumor volume, and adverse effects, in a series of 34 acromegalic patients, 32 of whom had macro- or invasive GH-secreting adenomas [12]. The study was performed in 
a single center, thus avoiding inter-center variability and enhancing the validity of the results. A total dose of $50 \mathrm{~Gy}$ was delivered in 27 separate sessions, five per week, to adenoma remnants that persisted after transsphenoidal neurosurgery. Mean follow-up after FSRT was 12 years and the patients were evaluated each year. The rate of hormonal remission, defined as a normal age-adjusted serum IGF-1 level without medical treatment for at least 3 months, gradually increased during follow-up, $50 \%$ of patients being free of medical treatment 15 years after FSRT. As observed with other modalities of pituitary radiotherapy, the remission rate was predicted both by circulating GH and IGF-1 levels and by tumor size prior to radiotherapy. Overall, the disease was controlled in $97 \%$ of patients at the end of the study. Patients who still needed medical therapy were able to receive lower drug dosages. Importantly, control of tumor volume was excellent, with stabilization of the residual tumor in $47 \%$ of patients and a size reduction in the remainder. Adverse effects were limited to pituitary deficiencies, observed in one-third of patients. Their late occurrence after FSRT means that each pituitary function should be monitored over the long term.

The results of this long-term study underline the value of FSRT as a third-line treatment for patients with $\mathrm{GH}$-secreting macro- or invasive adenomas. As expected, the success rate of FSRT increased gradually during follow-up but tended to plateau after about 15 years. FSRT thus holds promise as a further treatment option for patients with large $\mathrm{GH}$-secreting adenomas or adenomas too close to the optic chiasm, offering the combined advantages of stereotactic precision, limited adverse effects, and a reduction in costs associated with long-term medical treatment. Nevertheless, it remains to be seen whether this new technique (and radiosurgery too) will also help to avoid the cerebrovascular complications (and excess mortality) associated with "conventional" fractionated radiotherapy [13].

\section{Compliance with ethical standards}

Disclosure PC. has received unrestricted research and educational Grants from Ipsen, Novartis, Novo-Nordisk, and Pfizer at the Department of Endocrinology and Reproductive Diseases, Hôpitaux Universitaires Paris-Sud; he has served as an investigator (principal or coordinator) for clinical trials funded by Novartis, Pfizer, Ipsen, Italopharmaco, Antisense, Prolor Biotech; he is a member of the Advisory Boards from Ipsen, Novartis, Viropharma; also he gave lectures for Ipsen, Novartis, and Pfizer. All the fees and honoraria were paid to his Institution. EK. has no conflict of interest to disclose.

\section{References}

1. S. Melmed, Medical progress: acromegaly. N. Engl. J. Med. 355(24), 2558-2573 (2006)

2. J.A. Jane Jr, R.M. Starke, M.A. Elzoghby, D.L. Reames, S.C. Payne, M.O. Thorner, J.C. Marshall, E.R. Laws Jr, M.L. Vance, Endoscopic transsphenoidal surgery for acromegaly: remission using modern criteria, complications, and predictors of outcome. J. Clin. Endocrinol. Metab. 96(9), 2732-2740 (2011)

3. A. Giustina, P. Chanson, D. Kleinberg, M.D. Bronstein, D.R. Clemmons, A. Klibanski, A.J. van der Lely, C.J. Strasburger, S.W. Lamberts, K.K. Ho, F.F. Casanueva, S. Melmed, Expert consensus document: a consensus on the medical treatment of acromegaly. Nat. Rev. Endocrinol. 10(4), 243-248 (2014)

4. P. Chanson, S. Salenave, P. Kamenicky, Acromegaly. Handb. Clin. Neurol. 124, 197-219 (2014)

5. I. Yang, W. Kim, A. De Salles, M. Bergsneider, A systematic analysis of disease control in acromegaly treated with radiosurgery. Neurosurg. Focus 29(4), E13 (2010)

6. B.E. Pollock, How can fractionated radiotherapy and stereotactic radiosurgery be used for pituitary adenomas? Nat. Clin. Pract. Oncol. 4(12), 688-689 (2007)

7. J.S. Loeffler, H.A. Shih, Radiation therapy in the management of pituitary adenomas. J. Clin. Endocrinol. Metab. 96(7), 1992-2003 (2011)

8. S. Milker-Zabel, A. Zabel, P. Huber, W. Schlegel, M. Wannenmacher, J. Debus, Stereotactic conformal radiotherapy in patients with growth hormone-secreting pituitary adenoma. Int. J. Radiat. Oncol. Biol. Phys. 59(4), 1088-1096 (2004)

9. G. Minniti, D. Traish, S. Ashley, A. Gonsalves, M. Brada, Fractionated stereotactic conformal radiotherapy for secreting and nonsecreting pituitary adenomas. Clin. Endocrinol. 64(5), 542-548 (2006)

10. B.E. Pollock, J.T. Jacob, P.D. Brown, T.B. Nippoldt, Radiosurgery of growth hormone-producing pituitary adenomas: factors associated with biochemical remission. J. Neurosurg. 106(5), 833-838 (2007)

11. S. Roug, A.K. Rasmussen, M. Juhler, M. Kosteljanetz, L. Poulsgaard, H. Heeboll, H. Roed, U. Feldt-Rasmussen, Fractionated stereotactic radiotherapy in patients with acromegaly: an interim single-centre audit. Eur. J. Endocrinol. 162(4), 685-694 (2010)

12. A.M. Diallo, P. Colin, C.F. Litre, M.M. Diallo, B. Decoudier, F. Bertoin, B. Higel, M. Patey, P. Rousseaux, B. Delemer, Longterm results of fractionated stereotactic radiotherapy as third-line treatment in acromegaly. Endocrine (2015). doi:10.1007/s12020015-0610-1

13. M. Sherlock, J. Ayuk, J.W. Tomlinson, A.A. Toogood, A. Aragon-Alonso, M.C. Sheppard, A.S. Bates, P.M. Stewart, Mortality in patients with pituitary disease. Endocr. Rev. 31(3), 301-342 (2010) 